\title{
Development of Control Algorithm for Ring Laser Gyroscope
}

\author{
${ }^{1}$ P. Shakira Begum, ${ }^{2}$ N. Neelima \\ ', 2 Department of Electronics and communications Engineering, MRITS, JNTUH, HYDERABAD.
}

\begin{abstract}
RING LASER GYRO (RLG) is a sensor to sense angular information very precisely. It uses the principle of sagnac effect. In order to get excellent performance from a ring laser gyro, Intensity of laser beams, path length of the cavity and also the dithering mechanism needs to be ultra stable and maintained consistently. Hence control algorithms and hardware is implemented in RLGs for these three controls. Most of the algorithms implemented are linear algorithms and simple in nature. It is felt to use non linear algorithms based on the dynamics of the RLG to improve the performance of an $R L G$.

So in this project it is proposed to study the principles involved in an RLG control and simulate the dynamics for understanding the non-linearities and finally design new control algorithms for controlling RLG. $M A T L A B / L A B V I E W$ is proposed to be used for realizing these algorithms and finally implement them in DSP.

Keywords: $R L G, P L C, D P L C, P Z T, D A C$.
\end{abstract}

\section{Introduction}

A Ring Laser gyroscope is any device used to create a fixed direction in space or measure the angular rate and position of the platform to which it is mounted with respect to a fixed reference frame . RLG works on the principle of the Sagnac effect. The setup consists of a square or equilateral triangle shaped cavity with mirrors placed at corners. In this cavity, the laser medium is placed. The laser is used as a light source and the light generated travels in the cavity in two directions one in clockwise and the other one in counter clockwise. The output is obtained through one of the four mirrors which is partially transmitting to allow light through to a detector. The photo detector will detect the same interference pattern but with a $90^{\circ}$ phase difference. This arrangement is mounted on a rotating platform. When the beams are recombined, in the absence of rotation, the paths travelled by the two beams will be of same length.

However, if the apparatus is rotated, the beam that is travelling in the direction of rotation of the platform has a longer distance to travel (longer path length). Conversely, the beam travelling against the direction of motion has a shorter path length. So there will be a net path difference. This path length difference results in a phase difference which is directly proportional to amount of rotation. The net signal, i.e. the signal obtained by the interference of the two beams, will vary in amplitude depending on the phase shift, and consequently, the rotation rate. In order to get excellent performance from a ring laser gyro, Intensity of laser beams, path length of the cavity and also the dithering mechanism need to be ultra stable and maintained consistently.

\section{Path Length Control}

The RLG operates as a resonant cavity. The gas mixture, which sustains the laser, exhibits the gain at certain optical frequencies that excite the stimulated emission, resulting in lasing action. Therefore, the length of the cavity must be tuned to be an integer number of wavelengths. For a helium-neon gas mixture, the wavelength is approximately 630 nmeters. Obviously, a cavity whose length is accurate and stable to $1 \%$ of a wavelength would be impractical to design. Thus, cavity length is controlled actively by continuously adjusting mirror positions in order to maximize total laser intensity. Piezoelectric transducers mounted on the back of one or more mirrors induce minute displacements of the mirror faces .Since mirrors can move only a few wavelengths, the cavity must be made of a low-expansion glass so that the mirror travel is sufficient to compensate for expansion over the entire temperature range. Hence it is necessary to design an optimal control algorithm for controlling the path length. 


\section{Digital Path Length Control}

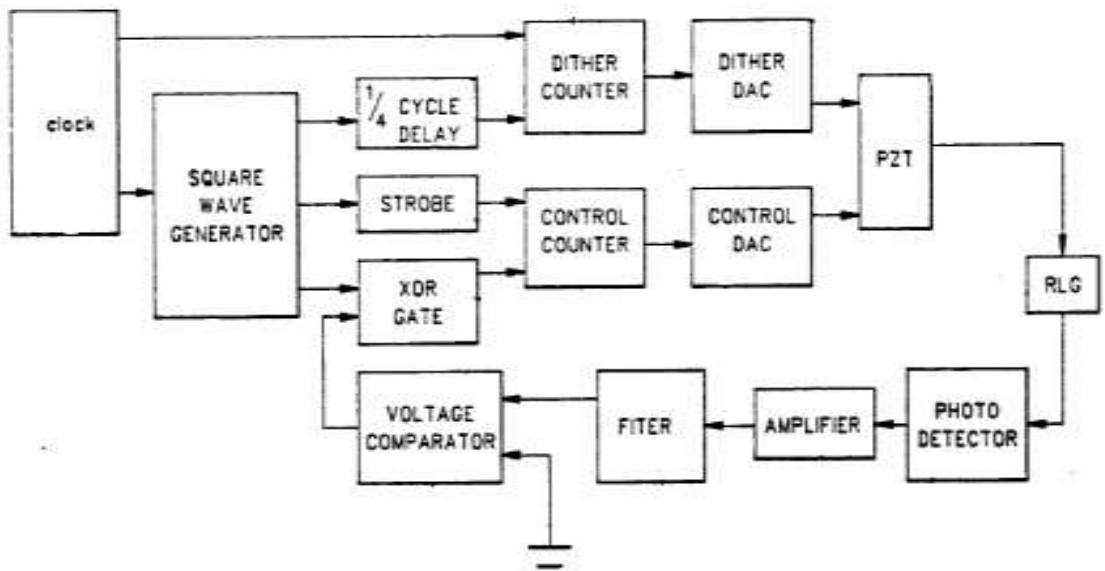

Fig 1.1 Digital path length control

A digital path length control(DPLC) for ring laser gyro comprises a square wave generator, a dither counter(driven by the generator with a quarter cycle delay), a dither DAC, a control counter, a control DAC, and a PZT driven by the two DAC's. The PZT controls the path length of the ring laser gyro, and thus controls the intensity of light in the gyro. A photodetector ground. The output of the comparator is XORed with the square wave, and the output of the XOR gate drives the control counter, strobed with a frequency at least twice that of square wave. All signal processing is digital, and analog conversion is made only when interfacing the gyro.

This method relates to means for controlling the path length of a ring laser gyro, and has particular relation to such means which are as completely digital as possible.

In a ring laser gyro(RLG), an optical ring is formed, and two laser beams are directed around the ring in opposite directions. When the beams are combined, rotation of the ring appears as an interference shift in the combined beams.

It is apparent that precise control must be maintained over the optical length of the path which the beams take around the ring. The conventional method is analog dithering. Referring now to Fig.1.2, the intensity of light produced by a laser is schematically plotted as a function of the path length of the RLG. At point 10, the path length is too short to produce its maximum output: at point 12 it is too long: and at point 14 it is just right.

\section{Method of controlling the path length}

The path length of an RLG can easily be controlled by controlling the position of one (or more) of mirrors which bounce the laser beam around the ring. This may most conveniently be accomplished by placing a piezoelectric transducer(PZT) on the back of the mirror, and controlling the thickness of the PZT by controlling the voltage which is fed to the PZT. If the RLG is operating at point 10, then the voltage to the PZT is increased: If the RLG is operating at point 12 , the voltage is decreased: and if the RLG is operating at point 14 , the voltage is kept constant. The PZT is constructed such that increasing the voltage makes the PZT thinner which(PZT is on the back of the mirror) increases the path length. Voltage polarity, PZT position and PZT operation(increased voltage makes it thicker) may be reversed in pairs if convenient.

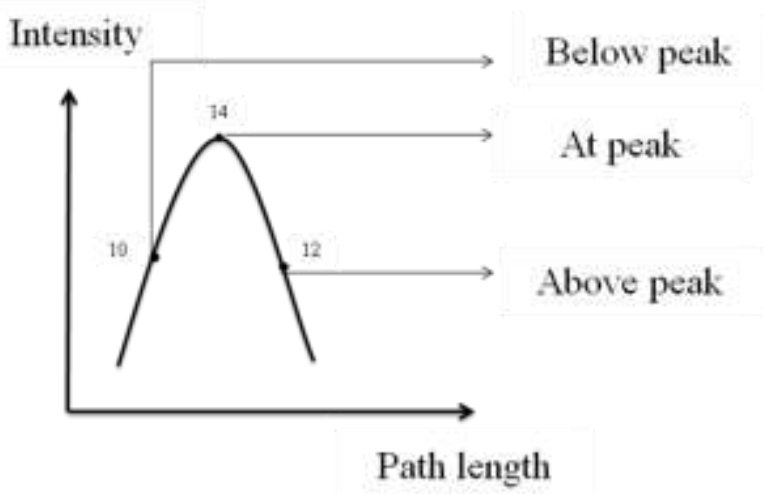

Fig.1.2 Path length Vs Intensity 
Dithering is used to determine the point at which the RLG is operating. Dithering is the application of a small AC sinusoidal voltage signal to the PZT causing the path length of the RLG to likewise vary sinusoidally. Turning now to Fig.1.3 the operation of dithering is shown. If the RLG was operating at point 10 without dithering, then it will operate at point 16 and point 18, and at every point in between, with dithering. If the RLG was operating at point 12 without dithering, it will instead operate between points 20 and 22, and if previously operating at point 14, it will now operate between points 24 and 26.

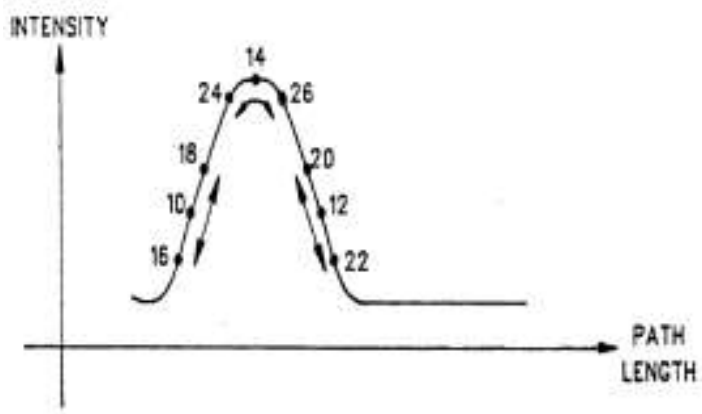

Fig.1.3 Effects of Dithering

Since the gain curve shown in above figures are relatively flat at the peak point 14 , in comparison with the sides 10 and 12, the amplitude of modulation of the light intensity is less when RLG is operating at its peak, in comparison to when it is operating with a path length which is either under or over the peak path length. The modulation at peak is therefore difficult to detect. Also at peak the peak intensity modulates with a frequency twice that of the dithering voltage.

The prior art uses analog components to form a phase comparator, into which is fed the dithering voltage and a pick-off voltage, that is the voltage produced by photodetector actuated by a small sample of light which has been picked off from the laser beam. If the two signals are in phase, then the voltage to the PZT is increased; if they are out of phase, it is decreased. An analog phase comparator and voltage feedback device suffers from the drawbacks of analog devices generally: radiation softness, bulk, weight, lack of tolerance for variation in component parameters, and the like. It is another objective to minimize the use of analog components.

It is a feature that it uses analog components only when interfacing with the laser beam, i.e., at the PZT and around the photodetector. The output from the photodetector is first amplified to a useful level, is then bandpass filtered to eliminate both inevitable noise which is present at frequencies higher than the highest frequency of interest(the modulation frequency when RLG is operating at peak) and any dc component, and is then immediately fed to specially designed analog to digital converter(ADC). Likewise, the PZT is driven directly by a digital to analog converter(DAC). It is an advantage that signal processing takes place between ADC and $\mathrm{DAC}$ and is entirely digital.

\section{Algorithm for Path Length Control} another.

Fig.1.1 shows in block form, the components of the apparatus and how they are connected to one

Step 1: The first necessity is to establish a digital dither signal. The most convenient way is with a counter, which increments by one each clock cycle when its control input is logic 0 , decrements by one when its control input is logic 1. As shown in the Fig1.1, a clock drives both a square generator and a dither counter. The dither counter is also driven by the square wave generator is first passed through a quarter cycle delay apparatus the purpose of which is described below. For as long as the output from the quarter cycle delay apparatus is logic 0 , the dither counter increments by one each clock cycle, and its output therefore shows a descending ramp. The clock signal itself is not shown since there should be a large multiplicity of clock pulses for each square wave pulse. For example a clock frequency can have $2^{13} * 1.5 \mathrm{KHz}$, while the square wave itself has a frequency of only $1.5 \mathrm{KHz}$. The large number of clock pulses cannot be shown and makes its presence felt in the smooth ramps rather than having these ramps being stair steps.

Step 2: The dither counter drives a dither DAC. This dither DAC in turn drives a PZT, which modulates the path length of the RLG. Since the dither DAC produces a triangular wave, the PZT also moves in a triangular wave rather than in a sine wave. The resulting modulation of the laser beams intensity is therefore also a triangular wave. The triangular wave is not only more easily produced than in a sine wave, it also has a more sharply defined peak than does a sine wave. The more sharply defined peak facilitates the task of actively controlling the path length of the RLG. 
Step 3: A photodetector samples a picked off portion of the laser beam propagating in RLG. This signal from photodetector is amplified by amplifier. As has been pointed out, the highest frequency of interest is the frequency detected by the photodetector when the RLG is operating at the peak intensity point 14 , i.e., twice the modulation frequency of the signal produced by the square wave generator. Noise is generally of higher frequency and is eliminated by a band-pass filter.

Step 4: Noise is generally of higher frequency and is eliminated by a band-pass filter. The low end of the bandpass filter may be conveniently set at one fifth of the modulation frequency of the signal produced by the square wave generator. This will eliminate any dc component without excessively affecting the square wave frequency. If desired, the band-pass filter may be replaced with a double band-pass filter, passing only the frequency produced by the square wave generator and twice that frequency. Such measures are generally not necessary.

Step 5: Now the output of the band-pass filter is fed to a voltage comparator. The voltage comparator compares the output of the filter with ground. It produces logic 1 when the filter output is positive with respect to ground and produces a logic 0 when the filter output is negative with respect to ground Since the filter output is centered on 0 volts, the voltage comparator produces a square wave. However the filter output is a triangular wave it crosses the zero voltage horizontal axis a quarter cycle out of phase with quarter cycle delay apparatus which ultimately produced it. However, it is exactly in phase with the output of the square wave generator which drives the quarter cycle delay apparatus. Thus, the square wave generated by the voltage comparator is exactly in phase with the square wave generated by the square wave generator.

Step 6: The square wave generator and the voltage comparator thus both produces digital square waves. These two signals are fed to the two inputs to an XOR gate. When these two signals are in phase, XOR gate produces a steady logic 0 which is shown in Fig.1.9. This summarizes the situation when the RLG is operating below peak path length. Also when these two signals are out phase, XOR gate produces a steady logic 1 which is shown in Fig.1.8. This summarizes the situation when the RLG is operating above peak path length and finally when comparator output is twice that of square wave, XOR gate produces a steady logic 0 and 1 which is shown in Fig.1.10 . This summarizes the situation when the RLG is operating at peak path length. All these operating conditions are depicted in the following diagrams with their waveforms.

Step 7: The feedback signal produced by the XOR gate drives a control counter, which decrements by one with each pulse from the strobe when the signal produced by XOR gate is a logic 0 and increments by 1 with each pulse when it is a logic 1 . This is the reverse of the operation of the dither counter as is to be expected in a negative feedback control loop.

Step 8: The control counter drives a control DAC which outputs its voltage to one of the terminals of the PZT. It will be recalled that the other terminal of the PZT id driven by the dither DAC. Here the thickness of the PZT (and consequent position of the mirror controlling the path length of the RLG) does not depend upon the absolute or average voltage impressed on its terminals, but on the voltage difference between them. Thus the single PZT responds both to the dither DAC and the control DAC.

Step 9: The control counter may be initialized such that the voltage produced by the control short, the XOR gate produces a logic 0 . This causes the control counter to decrement, which causes the output voltage of the control DAC to drop, which increases the voltage difference between output of control DAC and dither DAC. The PZT therefore gets thinner, increasing the path length of the RLG which is the desired result. Provided that the PZT is connected with the correct polarity, the same feedback will occur even when control DAC produces a voltage greater than that produced by the dither DAC. A similar feedback occurs when the path length is too long.

The above algorithm for three different conditions has been implemented in Xilinx 13.2i and the simulation output has been displayed below. Also this algorithm has been dumped into Vertex 4 evaluation platform.

The output waveforms of dither counter and control counter under three different peak conditions are shown below Fig 1.8 to Fig. 1.10

VI. Matlab Results

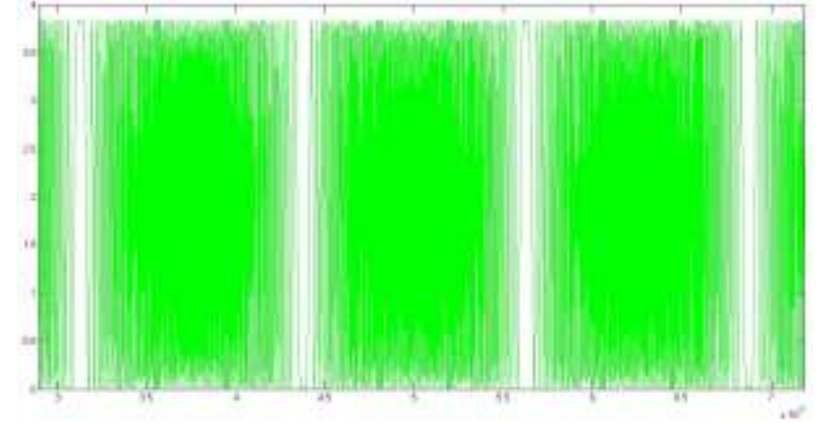




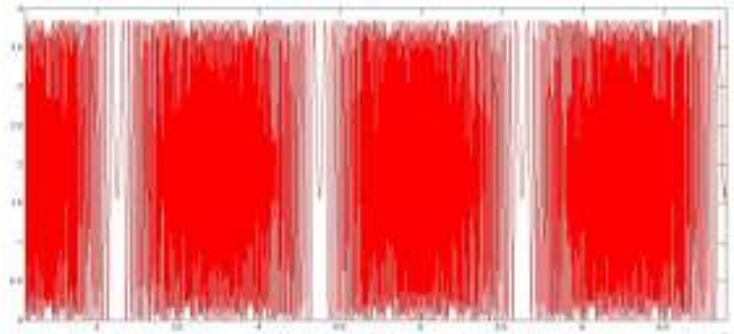

Fig.1.4 RLG output signals: Sin/Cos Signals

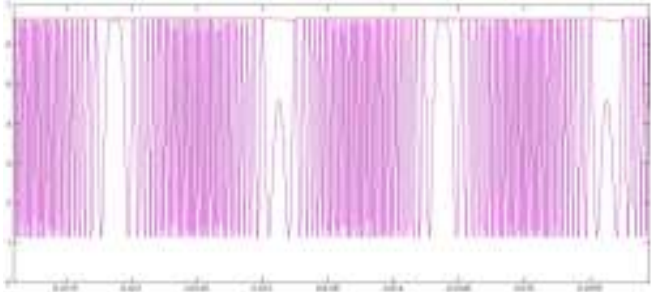

Fig.1.5 Peak Detection

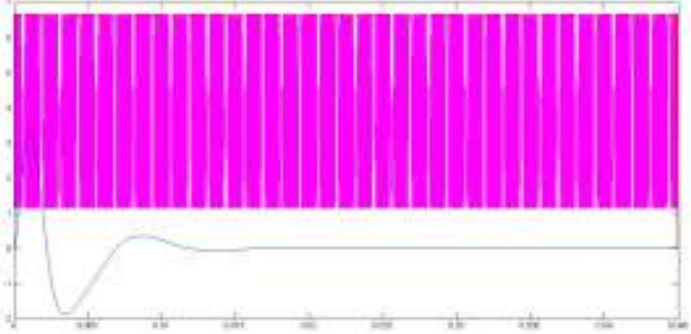

Fig.1.6 Band-pass Filtered output

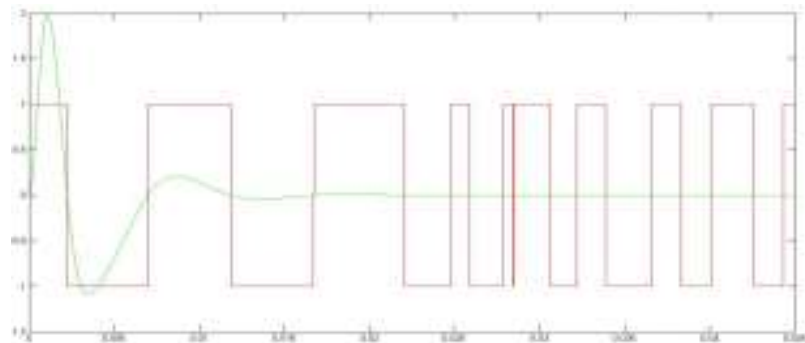

Fig.1.7 Converting to Square wave

VII. Xilinx Results

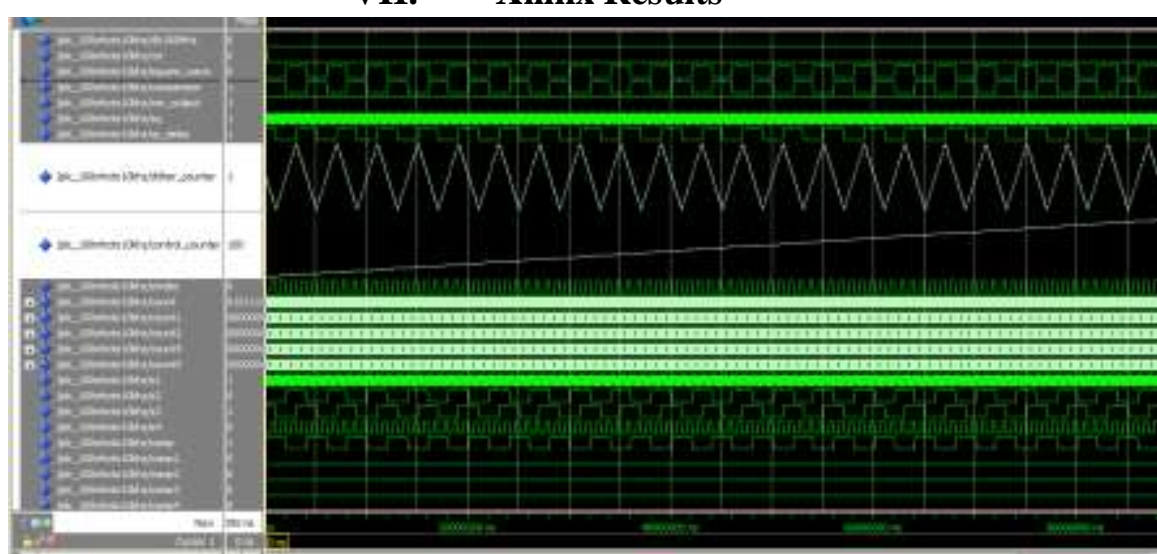

Fig 1.8 Above peak condition 


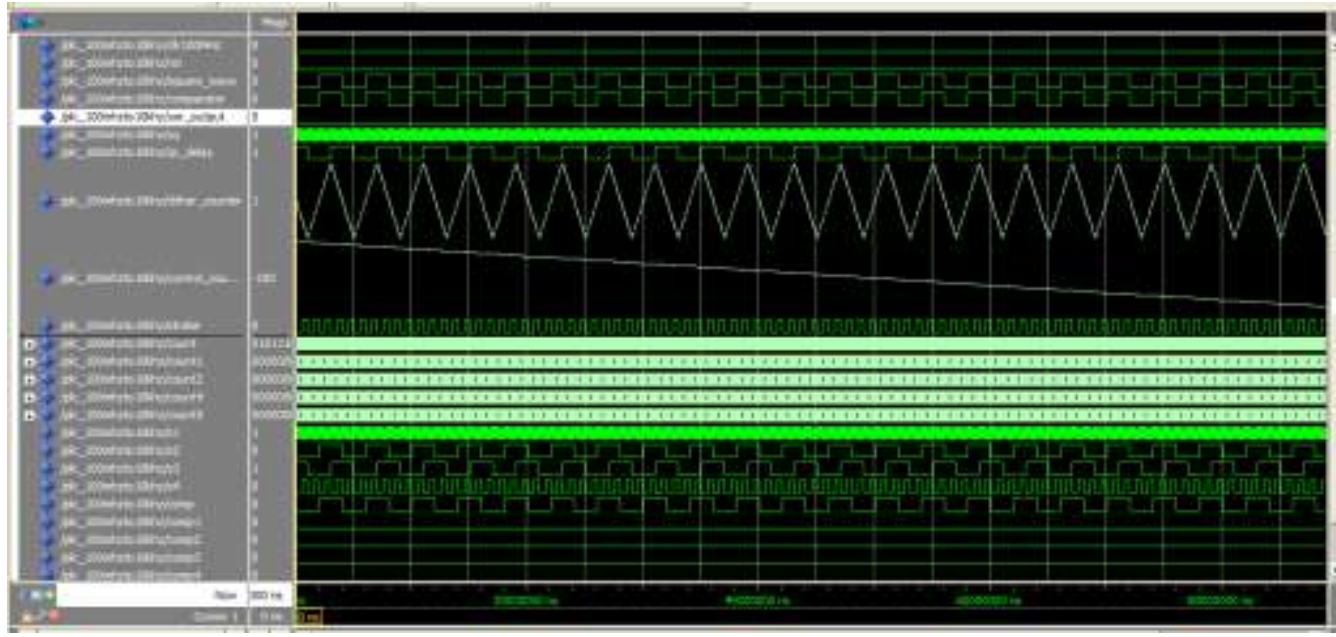

Fig.1.9 Below peak condition

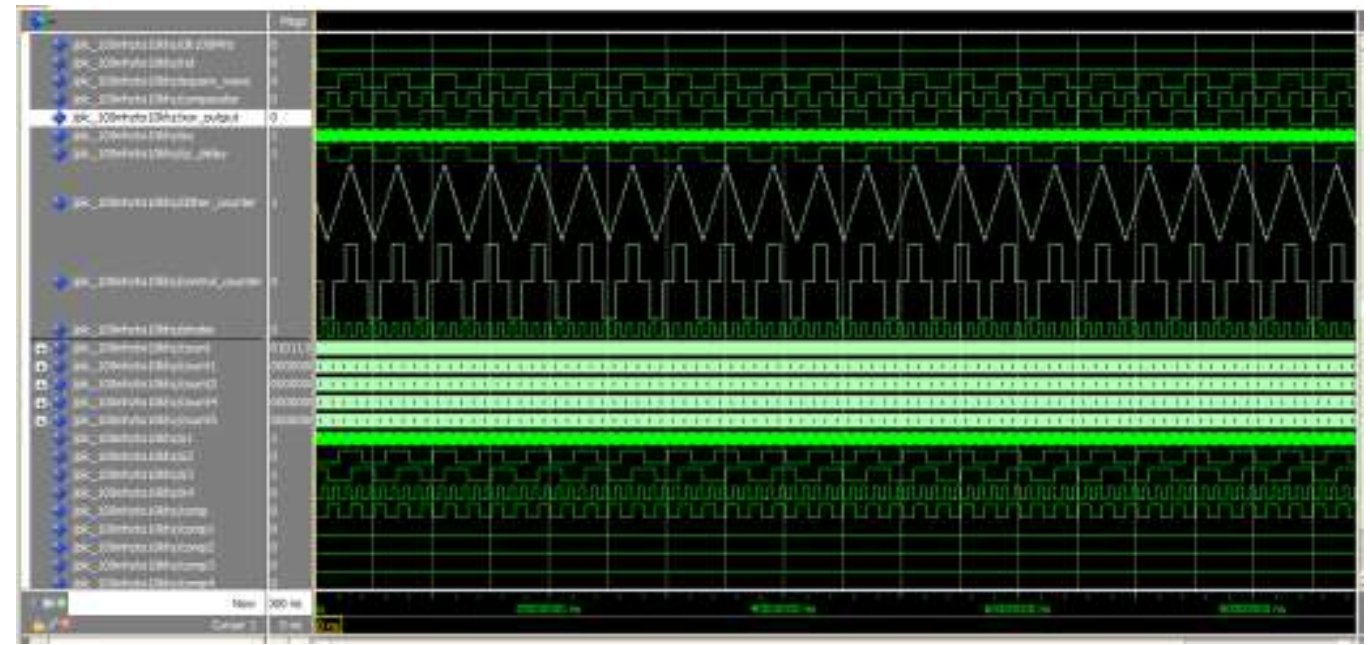

Fig.1.10 At peak condition

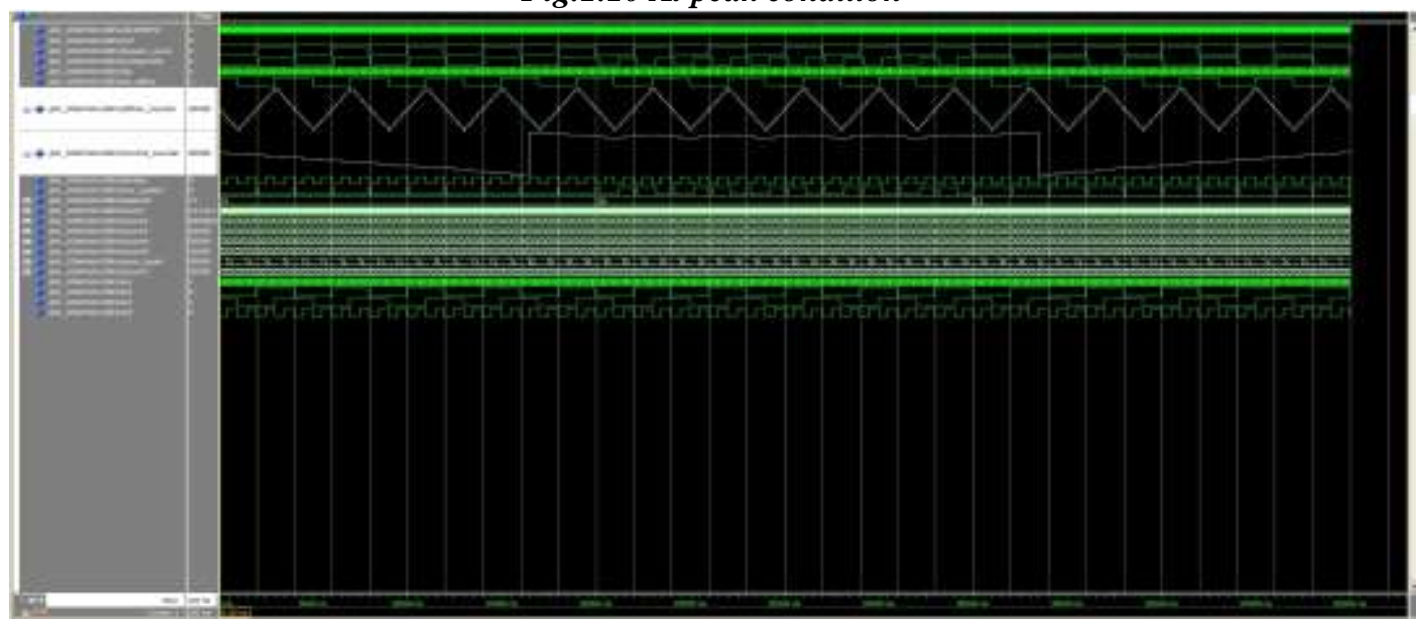

Fig.1.11 AllThree conditions

\section{Conclusion}

The objective of improving the performance of the Ring Laser Gyroscope was tackled by developing the control algorithms for the three functionalities that will govern the performance of the RLG. These three controls such as intensity of laser beams, path length of the cavity and also the dithering mechanism are controlled in such a way that they maintain consistently throughout the operation of RLG. RLGs have several benefits over conventional gyroscopes. For one, RLGs can be constructed to have no moving parts. Since RLGs 
do not rely on the conservation of angular momentum, they incur no resistance to rotation in sensitive systems where a conventional gyro might. The sensor output is inherently digital therefore easily incorporated into transistor-transistor logic systems.

\section{References}

[1] “Gyroscope.” McGraw-Hill Encyclopedia of Science and Technology. Vol. 8. 2002 ed.

[2] "Brief History of Gyroscopes." < http://einstein.stanford.edu/content/education/EducatorsGuide/Page30.html> 19 FEB 2005.

[3] "Heading Sensors." <http://www.eng.yale.edu/ee-labs/morse/other/pos96ch2.pdf>, 19 FEB 2005.

[4] Sharp, James. "Laser Gyroscopes." < http://www.mech.gla.ac.uk/ sharpj/lectures/lasers/notes/laser_gyro.pdf> 19 FEB 2005.

[5] "Ring Laser Gyroscopes" by Dr. James H. Sharp.

[6] G. L. Curran, and D. J. Engelken, "Ring laser gyro applications for tactical missiles: the Army TACMS solution", Position Location and Navigation Symposium, IEEE, 1990(3), pp. 543-548 